\title{
THE UNIFORMLY ACCELERATED STRING AND THE BELL SPACESHIP PARADOX
}

\author{
Miroslav Pardy \\ Department of Physical Electronics \\ Masaryk University \\ Kotlářská 2, 61137 Brno, Czech Republic \\ e-mail:pamir@physics.muni.cz
}

June 1, 2021

\begin{abstract}
We consider the string with the length $l$, the left end and the right end of which is non-relativistically and then relativistically accelerated by the constant acceleration $a$. We calculate the motion of the string with no intercalation of the Fitzgerald contraction of the string. We consider also the Bell spaceship paradox. The Bell paradox and our problem is in the relation with the Lorentz contraction in the Cherenkov effect (Pardy, 1997) realized by the carbon dumbbell moving in the LHC or ILC (Pardy, 2008). The Lorentz contraction and Langevin twin paradox (Pardy, 1969) is interpreted as the Fock measurement procedure (Fock, 1964;).
\end{abstract}

\section{Introduction}

We will consider the string with the length $l$, the left end of which is accelerated by the constant acceleration $a$ and the right end is accelerated also by the constant acceleration a. We will calculate the motion of such accelerated string.

The differential equation of motion of string element can be derived as it follows. We suppose that the force acting on the element $d l$ of the string is given by the law (Koshlyakov, et al., 1962):

$$
T(x, t)=E S\left(\frac{\partial u}{\partial x}\right)
$$

where $E$ is the modulus of elasticity, $S$ is the cross section of the string. We easily derive that 


$$
T(x+d x)-T(x)=E S\left(\frac{\partial u}{\partial x}\right)(x+d x)-E S\left(\frac{\partial u}{\partial x}\right)(x) \approx E S u_{x x} d x
$$

The mass $d m$ of the element $d l$ is $\varrho S d x$, where $\varrho$ is the mass density of the string matter and the dynamical equilibrium gives

$$
\varrho S d x u_{t t}=E S u_{x x} d x .
$$

Or, after minimal modification we get

$$
\frac{1}{c^{2}} u_{t t}-u_{x x}=0 ; \quad c=\left(\frac{E}{\varrho}\right)^{1 / 2} .
$$

The last procedure was performed evidently in order to get the wave equation.

\section{The non-relativistic acceleration of the string}

Our problem is described by the wave equation (Koshlyakov, et al., 1962)

$$
u_{t t}=c^{2} u_{x x}+g(x, t),
$$

where $g(x, t)=p(x, t) / \varrho, p(x, t)$ being a force and the boundary conditions are

$$
\begin{gathered}
u(x=0)=\kappa_{1}(t)=\frac{1}{2} a t^{2}, \\
u(x=l)=\kappa_{2}(t)=\frac{1}{2} a t^{2}+l=\kappa_{1}(t)+l .
\end{gathered}
$$

The initial conditions are

$$
u(t=0)=f(x) ; \quad u_{t}(t=0)=F(x) .
$$

The problem cannot be solved by the standard Fourier method because the boundary conditions (6)-(7) are not homogeneous. So, we introduce the auxiliary function (Koshlyakov et al., 1962)

$$
w(x, t)=\kappa_{1}(t)+\left[\kappa_{2}(t)-\kappa_{1}(t)\right] \frac{x}{l}
$$

with the boundary conditions

$$
w(x=0)=\kappa_{1}(t) ; \quad w(x=l)=\kappa_{1}(t)+l
$$

and we take the solution in the form:

$$
u(x, t)=v(x, t)+w(x, t) .
$$

with the boundary conditions

$$
v(x=0)=0 ; \quad v(x=l)=0
$$

and with the initial conditions

$$
v(t=0)=f_{1}(x) ; \quad v_{t}(t=0)=F_{2}(x) .
$$


After insertion of $u=v+w$ into ew (5), we get the following equation for $v$ and $w$ :

$$
v_{t t}=c^{2} v_{x x}+g(x, t)+c^{2} w_{x x}-u_{t t} .
$$

Then, if we use the definition of $w$ by eq. (9), we get equation for $v$ in the form:

$$
v_{t t}=c^{2} v_{x x}+g_{1}(x, t)
$$

where

$$
g_{1}(x, t)=g(x, t)-\kappa_{1}^{\prime \prime}(t)-\left[\kappa_{2}^{\prime \prime}(t)-\kappa_{1}^{\prime \prime}(t)\right] \frac{x}{l}
$$

So, we see, that the last algebraic procedures lead to new system of equations. Namely:

$$
v_{t t}=c^{2} v_{x x}+g_{1}(x, t)
$$

with

$$
v(x=0)=0 ; \quad v(x=l)=0
$$

and

$$
v(t=0)=f_{1}(x) ; \quad v_{t}(t=0)=F_{2}(x)
$$

It is easy to show that $g_{1}(x, t)=g-a$ and the system of equation to be solved is as follows:

$$
v_{t t}=c^{2} v_{x x}+g-a
$$

with

$$
v(x=0)=0 ; \quad v(x=l)=0
$$

and

$$
v(t=0)=f_{1}(x)=0 ; \quad v_{t}(t=0)=F_{1}(x)=0
$$

The solution of the system is well known (Koshlyakov et al., 1962) and so we write the final form:

$$
v(x, t)=\sum_{k=1}^{\infty} T_{k} \sin \left(\frac{k \pi x}{l}\right),
$$

where

$$
T_{k}(t)=\frac{2}{l \omega_{k}} \int_{0}^{t} d \tau \int_{0}^{l} G(\xi, \tau) \sin \omega_{k}(t-\tau) \sin \left(\frac{k \pi \xi}{l}\right) d \xi
$$

where

$$
\omega_{k}=\frac{k \pi c}{l} ; \quad G(\xi, \tau)=g-a .
$$

So, $u=v+w=v+\frac{1}{2} a t^{2}+x$. 


\section{The relativistic acceleration of the string}

Let us consider, at first, the relativistic uniformly accelerated motion of a particle, i.e. the rectilinear motion for which the acceleration $w$ in the proper reference frame (at each instant of time) remains constant.

In the reference frame where the particle velocity is $v=0$, the components of the fouracceleration is $a^{\prime}=\left(0, a / c^{2}, 0,0\right)$, where $a$ is the ordinary three-dimensional acceleration directed along the $x$ axis and $c$ is here the velocity of light. The relativistically invariant condition for uniform acceleration is the constancy of the four-scalar which coincides with $a^{2}$ in the proper reference frame (Landau, et al., 1987):

$$
-\frac{a^{2}}{c^{4}}=a^{i} a_{i} \equiv \text { const }
$$

In the "fixed" frame, with respect to which the motion is observed, writing out the expression for $a^{i} a_{i}$ gives the equation

$$
\frac{d}{d t} \frac{v}{\sqrt{1-\frac{v^{2}}{c^{2}}}}=a,
$$

or,

$$
\frac{v}{\sqrt{1-\frac{v^{2}}{c^{2}}}}=a t+\text { const }
$$

Setting $v=0$ for $t=0$, we find that const $=0$, so that

$$
v=\frac{v t}{\sqrt{1-\frac{a^{2} t^{2}}{c^{2}}}}=a t+\text { const },
$$

Integrating once more and setting $x=0$ for $t=0$, we find:

$$
x=\frac{c^{2}}{a}\left(\sqrt{1+\frac{a^{2} t^{2}}{c^{2}}}-1\right)
$$

For $a t \ll c$, these formulas go over the classical expressions $v=a t, x=a t^{2} / 2$. For at $\rightarrow \infty$, the velocity tends toward the constant value $c$.

The proper time of a uniformly accelerated particle is given by the integral

$$
\int_{0,}^{t} \sqrt{1-\frac{v^{2}}{c^{2}}}=\frac{c}{a} \sinh ^{-1} \frac{a t}{c}
$$

As $t \rightarrow \infty$ it increases much more slowly than $t$, according to the law $c / a \log (2 a t / c)$ (Landau, et al., 1987).

The solution is the same as in case of the non-relativistic motion, only with the replacing eqs. (6-7) by the equations with the relativistic motion, or with the boundary conditions

$$
\begin{gathered}
u(x=0)=\kappa_{1}(t)=\frac{c^{2}}{a}\left(\sqrt{1+\frac{a^{2} t^{2}}{c^{2}}}-1\right) \\
u(x=l)=\kappa_{2}(t)=\frac{c^{2}}{a}\left(\sqrt{1+\frac{a^{2} t^{2}}{c^{2}}}-1\right)+l=\kappa_{1}(t)+l
\end{gathered}
$$


and with the initial conditions

$$
u(t=0)=f(x) ; \quad u_{t}(t=0)=F(x) .
$$

The final solution of the system is the analogue of eqs. (23-25) (Koshlyakov et al., 1962). So, we write the final form:

$$
v(x, t)=\sum_{k=1}^{\infty} T_{k} \sin \left(\frac{k \pi x}{l}\right),
$$

where

$$
T_{k}(t)=\frac{2}{l \omega_{k}} \int_{0}^{t} d \tau \int_{0}^{l} \Gamma(\xi, \tau) \sin \omega_{k}(t-\tau) \sin \left(\frac{k \pi \xi}{l}\right) d \xi
$$

with

$$
\omega_{k}=\frac{k \pi(E / \varrho)^{1 / 2}}{l}
$$

and the function $\Gamma(\xi, \tau)$ is after some calculation given by the formula:

$$
\Gamma(\xi, \tau)=g-a\left(1+\frac{a^{2} \tau^{2}}{c^{2}}\right)^{-3 / 2} .
$$

\section{The Bell spaceship and the accelerated string}

The Bell thought experiment can be considered evidently as the analogue of our situation with the accelerated string. In the Bell version of the thought experiment (Bell, 1993), three spaceships $\mathrm{A}, \mathrm{B}$ and $\mathrm{C}$ are initially at rest in a common inertial reference frame, $\mathrm{B}$ and $\mathrm{C}$ being equidistant to $\mathrm{A}$. Then, a signal is sent from $\mathrm{A}$ to $\mathrm{B}$ and $\mathrm{C}$ int its simultaneously detected by B and $\mathrm{C}$, causing $\mathrm{B}$ and $\mathrm{C}$ starting to accelerate in the vertical direction with identical acceleration, while $\mathrm{A}$ stays at rest in its original reference frame.

Then, from the observer in A, - B, C, will have at every moment the same velocity, and so remain displaced one from the other by a fixed distance. Let fragile thread is tied initially between $\mathrm{B}$ and $\mathrm{C}$. Then, as the rockets speed up, it will become too short, because of the Fitzgerald length contraction, and must finally break. It must break when, at a sufficiently high velocity, the artificial prevention of the natural contraction imposes intolerable stress. According to Bell, there was "clear consensus" which asserted, incorrectly, that the string would not break.

However, Petkov (2009) and Franklin (2009) interpret this paradox differently. They agreed with the result that the string will break due to unequal accelerations in the rocket frames, which causes the rest length between them to increase. However, they denied the idea that those stresses are caused by length contraction. This is because length contraction has no "physical reality", but is the result of a Lorentz relativistic transformation of space and time which by itself can never cause any stress at all. Thus, the occurrence of such stresses in all reference frames is supposed to be the effect of relativistic acceleration alone.

So, we see that the verbal argumentation by Bell and Petkov and others is not unambiguous. In our case we use the mathematically rigorous argumentation and it means that what is calculated by us is the realistic behavior of accelerated string. 


\section{Discussion}

We have seen how to calculate the internal motion of the uniformly accelerated nonrelativistic and the relativistic string of the length $l$. The initial length of the string is in our calculation constant and every modification of length by the Fitzgerald contraction is not acceptable in the theory of the algorithm of calculation. So, the question arises if our mathematical approach is in contradiction with the Bell verbal argumentation.

Let us remark that the Bell problem can be related to the author investigation on Lorentz contraction in the Cerenkov effect (Pardy, 1997) due to the carbon dumbbell moving in the LHC or ILC (Pardy, 2008). The Lorentz contraction is not interpreted in the sense of the Fitzgerald contraction bat in the sense of the measurement procedure by Fock (1964). Such interpretation, where the relativity is interpreted in the sense of the measurement procedure of Fock was also used in the article on the Langevin twin paradox, or, in other words, on the clock paradox (Pardy, 1969).

\section{References}

Bell, J. S., Speakable and Unspeakable in Quantum Mechanics (Cam- bridge: Cambridge University Press, 1993) , pp 67-68

Fock, V. Theory of space, time and and gravitation, Second revised ed., (Pergamon Press, Oxford, London, ... 1964).

Franklin, J. (2010). Lorentz contraction, Bell's spaceships, and rigid body motion in special relativity, arXiv:0906.1919v3.

Koshlyakov, N. S., Gliner, E. V. and Smirnov, M. M. The Differential Equations of Mathematical Physics. (Nauka, Moscow, 1962). (in Russsian).

Landau, L. D. and Lifshitz, E. M. , The Classical Theory of Fields, Fourth revised English edition, (Amsterdam, Boston, Heidelberg, London, ..., 1987).

Pardy, M. (1969). A remark on the clock paradox, Phys. Lett. 28 A, No. 11, 766.

Pardy, M. (1997). The Čerenkov effect and the Lorentz contraction, Phys. Rev. A 55 (3) (1997) 1647-1652,

Pardy, M. (2008). The relativistic length contraction of the carbon dumbell moving in the LHC, or, ILC, arXiv: 0710.3489v2.

Petkov, V. (2009). Accelerating spaceships paradox and physical meaning of length contraction, arXiv:0903.5128v1. 